\title{
An investigation on the impact of carnosine on the myocardium in lower extremity ischemia-reperfusion injury in rats ir $^{2}$
}

\author{
Aydin Keskin b,1, Batur G Kanar ${ }^{\text {a, }, 1}$, Rayiha G Kanar ${ }^{\text {b,1 }}$, Alper Kepez ${ }^{\text {c,1 }}$, Bulent Tunerir ${ }^{\text {d,1 }}$ \\ a Department of Cardiology, Saureyyapasa Chest Disease and Thoracic Surgery Training and Research Hospital, Istanbul, Turkey \\ b Department of Cardiovascular Surgery, Van Training and Research Hospital, Van, Turkey \\ c Department of Cardiology,Marmara University, Istanbul, Turkey \\ d Depertmant of Cardiovascular Surgery, Osmangazi University, Eskisehir, Turkey
}

\section{A R T I C L E I N F O}

\section{Article history:}

Received 8 September 2016

Received in revised form 16 November 2016

Accepted 18 November 2016

Available online 29 December 2016

\begin{abstract}
A B S T R A C T
The purpose of this study was to investigate the effect of carnosine (CAR) on ischemia-reperfusion (I-R) injury to the myocardium occurring after occlusion reperfusion of the infrarenal abdominal aorta (IAA) in rats. In this study, 30 Sprague-Dawley rats were randomly divided into three equal groups which are control, I-R, and $\mathrm{I}-\mathrm{R}+\mathrm{CAR}$ groups. Laparotomy and IAA dissection were performed in all groups; occlusion was not performed in the control group. In the control group, the process was completed in $90 \mathrm{~min}$ as in the other two groups. In the I-R and I-R + CAR groups, the IAA was occluded by microvascular clamps; the first 30 min represented the ischemia period, followed by declamping and a 60 min reperfusion period. After the I-R period, rats were sacrificed. Heart specimens and blood samples were taken for histopathological and biochemical evaluation. Statistically significant alterations were observed in the control group in comparison with the other groups in the histopathological analyses. Although there was no significant difference between the I-R and I-R + CAR groups regarding the histopathological findings, the I-R + CAR group displayed more favorable histopathological findings compared with the I-R group. There were also significant alterations between the I-R and I-R + CAR groups regarding aspartate aminotransferase (AST), creatine kinase (CK), and lactate dehydrogenase (LDH) enzyme levels. The I-R + CAR group had significantly lower enzyme levels compared with the I-R group. In conclusion, carnosine seems to effectively prevent myocardial I-R injury in rats, as demonstrated by histopathological and biochemical evaluation.

(C) 2017 The Society of Cardiovascular Academy. Production and hosting by Elsevier B.V. All rights reserved. This is an open access article under the CC BY-NC-ND license (http://creativecommons.org/licenses/by-nc-nd/4.0/).
\end{abstract}

\section{Introduction}

It is well established that deprivation of intracellular enzymes, antioxidants, and homeostatic mechanisms occur as a result of ischemia associated with reduced arterial or venous blood flow. ${ }^{1}$ Reperfusion, the only treatment available for ischemia, increases ischemia-mediated tissue injury, thereby leading to an extension of the infraction site. This process is called "reperfusion injury".2 The most significant mechanism involved in the reperfusion process is the direct toxic effect of the free oxygen radicals, originating from proinflammatory cells. ${ }^{3}$ Free radicals exert their biological effects by acquiring electrons from molecules, including lipids and proteins. Endogenous antioxidant defense systems,

\footnotetext{
it Peer review under responsibility of The Society of Cardiovascular Academy.

* Corresponding author at: Department of Cardiology, Sureyyapasa Chest Disease and Thoracic Surgery Training and Research Hospital, Basıbuyuk Mah. Maltepe, Istanbul, Turkey.

E-mail address: baturkanar@hotmail.com (B.G. Kanar).

1 Equal contributors.

Peer review under responsibility of The Society of Cardiovascular Academy.
}

such as superoxide dismutase, catalase, glutathione peroxidase, glutathione and coenzyme Q10 (ubiquinone) are used in cardiomyocytes. Myocardial stunning, necrosis, apoptosis, and vascular dysfunction may occur as a result of this process ${ }^{4,5}$.

Lower extremity ischemia-reperfusion (I-R) is a common event with clinically significant outcomes. Following ischemia, local and systemic injury starts due to the effect of oxygen radicals and inflammatory mediators, together with reperfusion. While restoration of blood flow saves the extremity, it may also lead to multisystem organ dysfunction and mortality. Local effects are observed in the skeletal muscle and vascular endothelium, while systemic effects occur particularly in the myocardial tissue, lungs, and kidneys ${ }^{6}$.

Carnosine (CAR; $\beta$-alanyl-L-histidine) is a biologically active dipeptide. It is synthesized from $\beta$-alanine an L-histidine by carnosine synthase in the organism. It has been reported to act as a physiological buffer. CAR has been demonstrated to act as a "scavenger" for free radicals and aldehydes and to suppress oxidative changes in proteins. ${ }^{7}$ Moreover, it has been reported to prevent the formation of advanced glycation end products and form complexes with bivalent metal ions. 
It has been suggested that CAR might be an effective agent in avoiding certain pathological conditions associated with oxidative stress such as aging, Alzheimer's disease, atherosclerosis, and liver injury. ${ }^{8}$

Today, despite the advanced opportunities offered by modern cardiovascular surgical methods and techniques, I-R following aortic procedures remains a serious issue that affects postoperative morbidity and mortality. Acute ischemia of skeletal muscles is a highly common condition. Particularly in case of aortic surgery, this occurs as a result of aortic cross-clamping. The risk of morbidity and mortality remains even after the cause of the acute ischemic condition is eliminated and reperfusion is achieved. ${ }^{9}$

Relevant studies have investigated the protective effect of CAR in the case of skeletal muscle I-R injury and obtained favorable results. ${ }^{10,11}$ However, data related with the impact of carnosine on abdominal aortic I-R injury and distant organ injury is limited. In our study, we intended to investigate the protective effects CAR on the myocardium following lower extremity I-R injury.

\section{Material and method}

The study was initiated after approval from the Animal Studies Local Ethical Committee of Eskisehir Osmangazi University (dated 17.10.2012, numbered 295/2012). Thirty Sprague-Dawley rats (both genders) with a mean weight of 250-300 g were randomized into three groups which are control, I-R, and I-R + CAR groups $(n=10$ each). All rats received $50 \mathrm{mg} / \mathrm{kg}$ of ketamine hydrochloride anesthesia via the intramuscular route following $8 \mathrm{~h}$ of fasting. An atraumatic microvascular clamp was placed in the infrarenal abdominal aorta (IAA) after laparotomy (Novaclip ${ }^{\circledR} 12$ mmangle) in all rats. The abdomen was sutured to reduce heat and fluid losses. Following ischemia for $30 \mathrm{~min}$, reperfusion was administered for $1 \mathrm{~h}$. Rats in the control group underwent laparotomy and IAA dissection for an equal period of time; however, this group did not undergo I-R. In the carnosine group, silk sutures were removed 10 min before completion of the ischemia period and removal of the clamp, and intraperitoneal CAR ( $\mathrm{L}-$ carnosine, Sigma-Aldrich, CAS no: 305-84-0) at a dose of $250 \mathrm{mg} / \mathrm{kg}$ was administered; normal saline perfusion was given in the other groups. At the end of the experiment, rats in all groups were sacrificed via administration of a high dose of anesthesia; $5 \mathrm{~cm}^{3}$ of blood was withdrawn from the aorta and the heart tissues were extracted and stored in $10 \%$ formaldehyde solution. Hearts from each groups were excised and fixed in $4 \%$ paraformaldehyde overnight at $4{ }^{\circ} \mathrm{C}$, subsequently dehydrated, and embedded in paraffin. Hearts from longitudinal or transverse sections sliced at 5-mm thickness were fixed on Superfrost Plus glass slides (Fisher Scientific, Toronto, ON, Canada). The sections were deparaffinated and stained with hematoxylin and eosin (H\&E stain) followed by a graded dehydrated alcohol and then mounted by Cytoseal XYL (Thermo Scientific, Waltham, MA). The images were visualized under a microscope and analyzed with Scope Image software version 9.0 (United Scope Inc., Kitchener, ON, Canada).

\section{Statistical analysis}

All study data were analyzed using PASW Statistics 18.0 and SigmaStat 3.5 packaged software. Continuous quantitative data were expressed in terms of $n$, mean, and standard deviation, while qualitative data were expressed in terms of $n$, median values, and 25th and 75th percentiles. Continuous data that consisted of independent measures
A
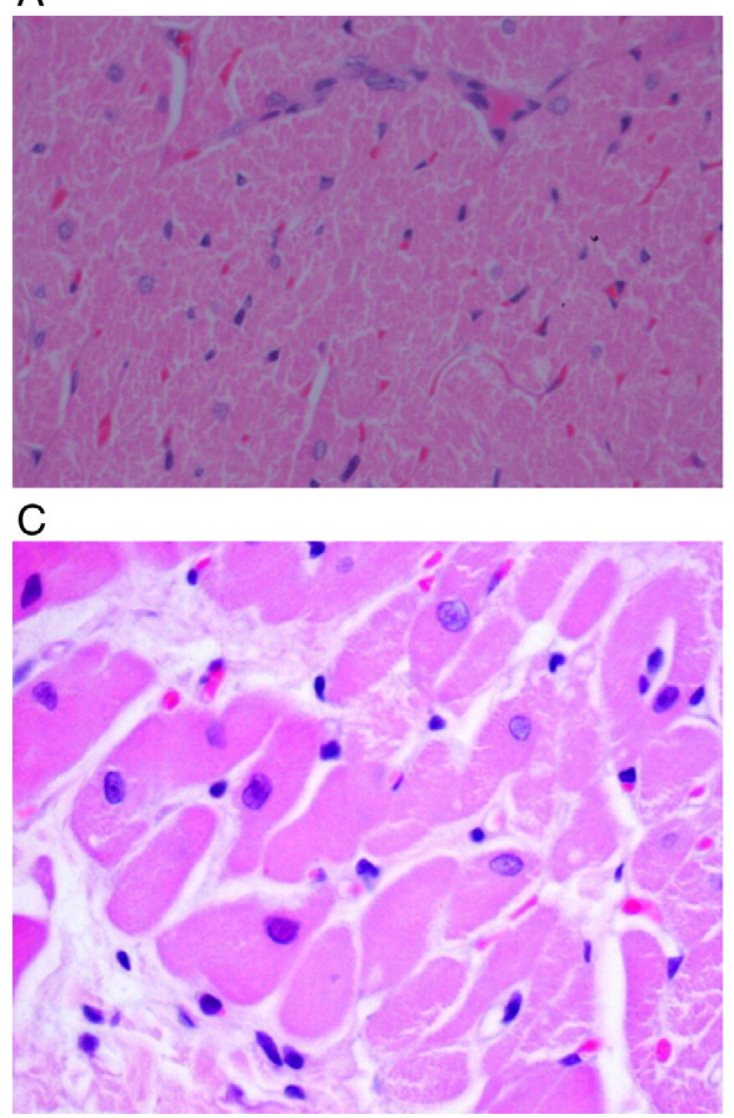

B

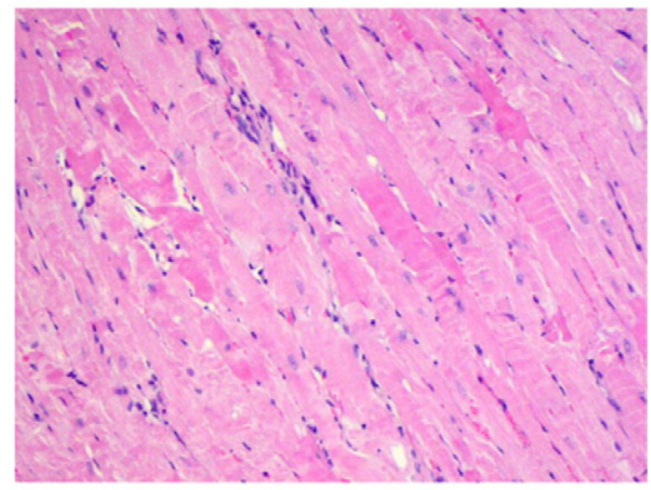

D

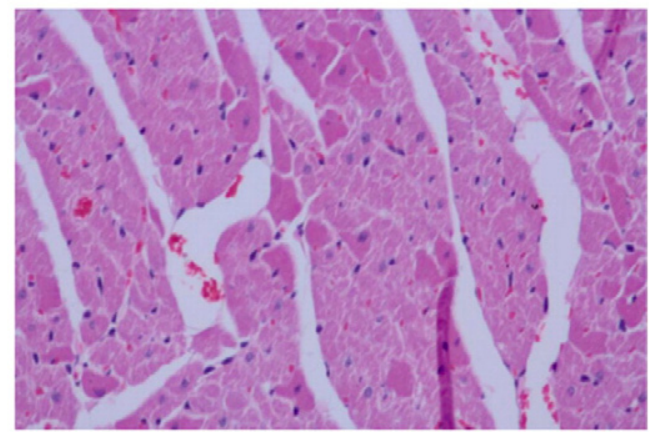

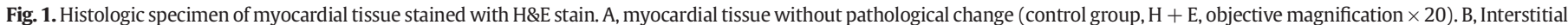

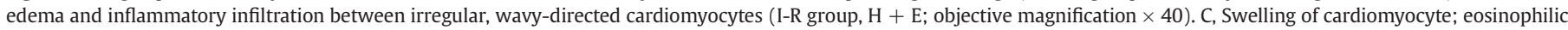

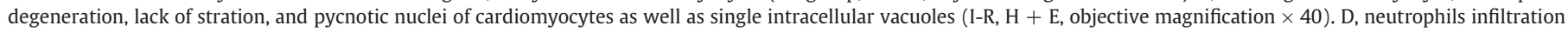
(Original magnification, $\times 20$ ). 
and exhibited normal distribution were analyzed using one-way analysis of variance (ANOVA). Furthermore, the Kruskal-Wallis test was used to analyze data consisting of score variables without normal distribution by group number. Finally, $p<0.05$ probability values were considered significant.

\section{Results}

In our study, all myocardial tissues were classified according to their histopathological features by light microscope. Histopathological classification included interstitial edema (Fig. 1b), swelling of cardiomyocytes (Fig. 1c), and neutrophil infiltration (Fig. 1d). Histopathological examination for all groups is reported in Table 1. Our control group was significantly different from the I-R and I-R + CAR groups (Table 2). There was no statistically difference between the I-R and I$\mathrm{R}+$ CAR groups.

To determine myocardial damage, we used plasma creatine kinase (CK), aspartate aminotransferase (AST), and lactate dehydrogenase (LDH) levels. In our control group, we took blood samples in the same time period as for the other investigation groups. Statistical analyses of the three groups are given in Tables 3 and 4 . To summarize, the I-R and I-R + CAR groups were statistically significantly different from the control group for all blood samples. However, there was a statistically difference between the I-R and I-R + CAR groups in the AST and LDH levels.

\section{Discussion}

I-R injury is the most common type of cell injury in clinical cases including coronary artery disease, coronary artery bypass grafting, fibrinolytic treatment, balloon angioplasty, obstructive arterial disorders, stroke, myocutaneous tissue transfer and re-implantation procedures. ${ }^{12}$ Intracellular enzymes, antioxidants, and homeostatic mechanisms are known to be impaired with ischemia. ${ }^{1}$ The most significant mechanism involved in the reperfusion process is the direct toxic effect of the free oxygen radicals originating from proinflammatory cells. Free radicals exert their biological effects by acquiring electrons from molecules including lipids, proteins, and deoxyribonucleic acid (DNA). Free radicals initiate lipid peroxidation, which acts on the cell membrane. In cardiomyocytes, there are endogenous antioxidant defense systems, including those of superoxide dismutase, catalase, glutathione peroxidase, glutathione, and coenzyme Q10 (ubiquinone). ${ }^{4}$ Excessive free radical formation that overwhelms the antioxidant defense systems may lead to myocardial stunning, necrosis, apoptosis, vascular dysfunction, dysrhythmia, and cardiac failure. ${ }^{9,13}$ Myocardial stunning, which is usually characterized by contractile dysfunction following I-R injury, may require the use of inotropic drugs and mechanical circulation support. ${ }^{14}$

Interventions aimed at reducing or preventing I-R injury consist of various procedures and medications. Such methods include antioxidant treatment (using superoxide dismutase, $\mathrm{N}$-acetyl cysteine, allopurinol, catalase, vitamin E, thiol, and mannitol), calcium antagonists, angiotensin-converting enzyme inhibitors, anti-complement treatment, leukocyte filtration, anti-cytokine adhesion molecules, endothelin receptor antagonists, platelet activating factor (PAF) antagonists, leukotriene B4 (LTB4) antagonists and procedures like controlled reperfusion, acute intermittent hypoxia, and ischemic preconditioning ${ }^{14,15}$.

Carnosine, a dipeptide ( $\beta$-alanine- $\mathrm{L}$-histidine), is present in the cardiac muscle cell at a level of $2-10 \mathrm{mM}$, which is present at highest concentrations in the skeletal muscle. Muscle carnosine is a major contributor to hidrogen buffering during high-intensity exercise. ${ }^{16}$ preliminary studies discovered that power athletes have higher carnosine levels than untrained individuals and endurance athletes. Moreover, Lombardi et al. showed that the oral supplementation of L-carnosine increases the quality of life and exercise performance in patients with stable chronic heart failure. ${ }^{17}$ The CAR tissue level is known to be

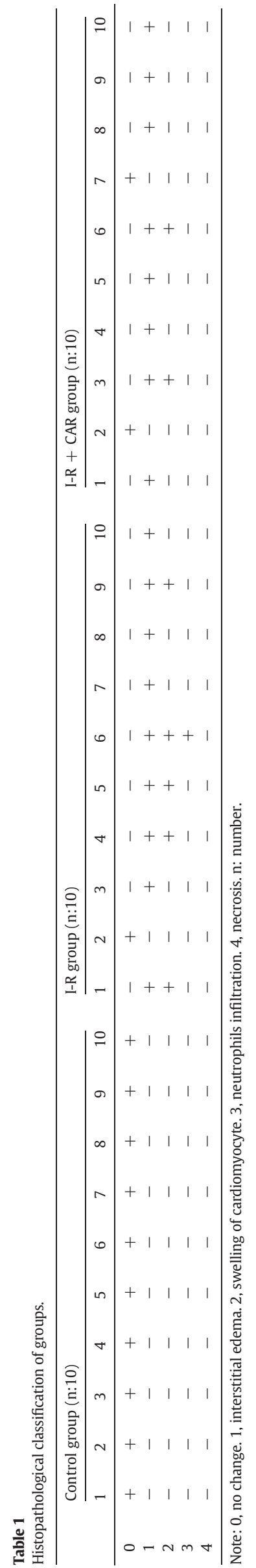


Table 2

Comparison of all groups with Kruskall-Wallis test.

\begin{tabular}{|c|c|c|c|}
\hline Histopathology & Median & $(25-75)$ & $\mathrm{p}$ \\
\hline Control (n:10) & 0.00 & $(0.00-0.00)$ & $\mathrm{p}<0.001^{/ 4}$ \\
\hline I-R (n:10) & 1.50 & $(1.00-2.00)$ & \\
\hline $\mathrm{I}-\mathrm{R}+\mathrm{CAR}(\mathrm{n}: 10)$ & 1.00 & $(1.00-1.00)$ & \\
\hline
\end{tabular}

$\gamma$ : statistical comparison of control and I-R groups. ${ }^{\phi}$ : statistical comparison of control and I$\mathrm{R}+\mathrm{CAR}$ groups.

decreased in animals with chronic infection. ${ }^{18}$ In addition, the tissue level of CAR also decreases during trauma in conditions that impair cardiac contractility. ${ }^{19}$ In the presence of weak alkaline $\mathrm{pH}, \mathrm{CAR}$ inhibits lipid peroxidation ${ }^{20}$.

Given its antioxidant efficacy, the impact of CAR has been investigated in many experimental models. In a rabbit model in which HF was induced by infusing doxorubicin, the administration of carnosine reduced cardiotoxic effects compared with rabbits treated with doxorubucine alone. ${ }^{21}$ Stvolinsky et al. ${ }^{22}$ showed that CAR had a neuroprotective effect in subjects with induced cerebral ischemia, and in another trial, they showed that administration of CAR extended the lifespan in subjects with induced global cerebral ischemia. Fujii et al. ${ }^{23}$ reported that administration of CAR reduced sympathetic nerve activity and I-R injury occurring in the kidneys in their renal $I-R$ model. Lee et al. ${ }^{24}$ demonstrated the protective effect of CAR against reperfusion injury in the isolated rat heart. In our trial, we induced an empirical I-R model via IAA occlusion in rats and investigated the effect of CAR on distant organ (myocardium) injury.

Shiny et al. ${ }^{25}$ showed the pro-oxidant effect of taurine through the reduction observed in the activities of plasma ALT, AST, LDH and CK-which are assessed clinically on a routine basis-in rats with myocardial infraction induced by isoprenaline administration. Panda et al. ${ }^{26}$ showed the effects of isoproterenol on myocardial injury via AST, LDH, and CK enzyme levels. They detected low enzyme levels in the group receiving ginkgo biloba and Ocimum sanctum in combination as an antioxidant. In our trial, we measured CK, AST, and LDH enzyme levels as the determinants of myocardial injury in the blood samples of rats and found that the enzyme levels were significantly low in the I-R + CAR group $(p<0.001)$.

Courten et al. showed that two-hour glucose and insulin were both lower after carnosine supplementation compared to placebo in individuals with impaired glucose tolerance. ${ }^{27}$

\section{Conclusion}

Early diagnosis and revascularization is of high importance in reducing lower extremity I-R injury and minimize local and distant effects. In addition, elucidation of the mechanism responsible for I-R injury would ensure rapid and effective blocking of the injury.

In our study, we showed CAR to be an effective agent in avoiding myocardial I-R injury, particularly on a biological basis. Even if its role in reducing I-R injury was not to be found statistically significant in histopathological assessment, we considered the detection of lower histopathological changes in the I-R + CAR group relative to I-R group to be significant. The reason for the failure to obtain a statistically significant

Table 3

Statistical analysis of CK blood sample by Kruskall-Wallis test.

\begin{tabular}{llll}
\hline CK & Median & $(25-75)$ & $\mathrm{p}$ \\
\hline Control $(\mathrm{n}: 10)$ & 2979.50 & $(2841.00-3341.00)$ & $\mathrm{p}<0.001^{\text {兰 }}$ \\
I-R (n:10) & 6566.50 & $(4548.00-7018.00)$ & \\
$\mathrm{I}-\mathrm{R}+$ CAR(n:10) & 3889.00 & $(3387.00-4126.00)$ & \\
\hline
\end{tabular}

${ }^{\gamma}$ : statistical comparison of control and I-R groups. ${ }^{\phi}$ : statistical comparison of control and I$\mathrm{R}+$ CAR groups
Table 4

Statistical analysis of AST and LDH blood samples by One-way ANOVA test.

\begin{tabular}{lll}
\hline & $\mathrm{X} \pm \mathrm{SD}$ & $\mathrm{p}$ \\
\hline AST & & $\mathrm{p}<0.001^{1 \notin 3}$ \\
Control (n:10) & $269.20 \pm 43.04$ & \\
I-R (n:10) & $448.10 \pm 49.23$ & \\
I-R + CAR (n:10) & $344.70 \pm 46.57$ & \\
LDH & & \\
Control (n:10) & $5298.40 \pm 258.99$ & \\
I-R (n:10) & $6081.80 \pm 324.27$ & \\
I-R + CAR (n:10) & $5487.30 \pm 322.33$ & \\
\hline
\end{tabular}

SD: standard deviation. ${ }^{\gamma}$ : statistical comparison of control and I-R groups. ${ }^{\phi}$ : statistical comparison of control and I-R + CAR groups. 3: statistical comparison of I-R and I$\mathrm{R}+$ CAR groups.

result may have been our small sample size. Thus, we consider that our study may guide more comprehensive future research.

\section{Disclosure of conflict of interest}

None.

\section{References}

1. Vermeiren GLJ, Claeys MJ, Van Bockstaele D, et al. Reperfusion injury after focal myocardial ischaemia: polymorphonuclear leukocyte activation and its clinical implications. Resuscitation 2000;45(1):35-61.

2. Braunwald EKR. Myocardial reperfusion: a double edged sword? J Clin Invest 1985;76:1713-1719.

3. Vinod NKRS, Murugesan C, Muralidhar K. Myocardial ischaemic pre-conditioning Indian J Anaesth 2004;48(2):93-99.

4. Kevin LG, Novalija E, Stowe DF. Reactive oxygen species as mediators of cardiac injury and protection: the relevance to anesthesia practice. Anesth Analg 2005;101(5): $1275-1287$.

5. Maxwell SRJ, Lip GYH. Reperfusion injury: a review of the pathophysiology, clinical manifestations and therapeutic options. Int J Cardiol 1997;58(2):95-117 (1/31/).

6. Blaisdell FW. The pathophysiology of skeletal muscle ischemia and the reperfusion syndrome: a review. Cardiovasc Surg 2002;10(6):620-630 (12//).

7. Boldyrev AA. Protection of proteins from oxidative stress: a new illusion or a novel strategy? Ann N Y Acad Sci 2005;1057(1):193-205.

8. Aldini G, Facino RM, Beretta G, et al. Carnosine and related dipeptides as quenchers of reactive carbonyl species: from structural studies to therapeutic perspectives. Biofactors 2005;24(1-4):77-87.

9. Baxter GF. The neutrophil as a mediator of myocardial ischemia-reperfusion injury: time to move on. Basic Res Cardiol 2002;97:268-275.

10. Fujii T, Takaoka M, Muraoka T, et al. Preventive effect of l-carnosine on ischemia/reperfusion-induced acute renal failure in rats. Eur J Pharmacol 2003;474(2-3):261-267 (8/8/)

11. Stvolinsky SLKM, Dobrota D, Matejovicova M, et al. Carnosine: an endogenous neuroprotector in the ischemic brain. Cell Mol Neurobiol 1999;Feb(19):45-46.

12. Atahan E, Ergun Y, Belge Kurutas E, et al. Ischemia-reperfusion injury in rat skeletal muscle is attenuated by zinc aspartate. J Surg Res 2007;137(1):109-116 (1//).

13. Aiello EAJR, Cole WC. Arrhythmia and delayed recovery of cardiac action potential during reperfusion after ischemia role of oxygen radical-induced no-reflow phenomenon. Circ Res 1995;77:153-162.

14. Collard CDGS. Pathophysiology, clinical manifestations and prevention of ischemia-reperfusion injury. Anesthesiology 2001;94:1133-1138.

15. Maxwell SLG. Reperfusion injury: a review of the pathophysiology, clinical manifestations and therapeutic options. Int J Cardiol 1997;58:95-117.

16. Parkhouse WS, McKenzie DC, Hochachka PW, et al. Buffering capacity of deproteinized human vastus lateralis muscle. J Appl Physiol 1985;58(1):14.

17. Lombardi C, Carubelli V, Lazzarini V, et al. Effects of oral administration of orodispersible levo-carnosine on quality of life and exercise performance in patients with chronic heart failure. Nutrition 2014;31(1):72-78.

18. Fitzpatrick DAJ, Squibb RL, Fisher $\mathrm{H}$. Effects of chronic and acute infections on tissue levels of carnosine, anserine and free histidine in rats and chickens. Proc Soc Exp Biol Med 1980;165:404-408.

19. Fisher DE AJ, Strumeyer DH, Fisher H. A role for carnosine and anserine in histamine metabolism of the traumatized rat. Proc Soc Exp Biol Med 1978;158:402-405.

20. Herman EHFV. Preclinical animal models of cardiac protection from anthracycline-induced cardiotoxicity. Semin Oncol 1998;25:15-21.

21. Zieba RW. Influence of carnosine on the cardiotoxicity of doxorubicin in rabbits. Pol J Pharmacol 2003;55:1079-1087.

22. Stvolinsky SL, Dobrota D, Mezesova V. Carnosine protects rats under global ischemia. Brain Res Bull 2000;53(4):445-448.

23. Fujii TTM, Muraoka T, Kurata H, et al. Preventive effect of $\mathrm{L}$ - carnosine on ischemia/reperfusion - induced acute renal failure in rats. Eur J Pharmacol 2003;8: 261-267. 
24. Lee JWMH, Bobst EV, Hester JD. Improved functional recovery of ischemic rat hearts due to singlet oxygen scavengers histidine and carnosine. J Mol Cell Cardiol 1999;31: 113-121.

25. Shiny KSKS, Farvin KHS, Anandan R, et al. Protective effect of taurine on myocardial antioxidant status in isoprenaline-induced myocardial infarction in rats. J Pharm Pharmacol 2005;57:1-5.
26. RN PVaS. Evaluation of cardioprotective activity of ginkgo biloba and ocimum sanctum in rodents. Altern Med Rev 2009;14(2):161-171.

27. de Courten B, Jakubova M, de Courten MPJ, et al. Effects of carnosine supplementation on glucose metabolism: pilot clinical trial. Obesity 2016;24(5):1027-1034. 